\title{
PERMANENT VERSUS DETERMINANT OVER A FINITE FIELD
}

\author{
G. Dolinar, A. Guterman, B. Kuzma, and M. Orel \\ UDC 512.5
}

\begin{abstract}
Let $\mathbb{F}$ be a finite field of characteristic different from 2 . We study the cardinality of sets of matrices with a given determinant or a given permanent for the set of Hermitian matrices $\mathcal{H}_{n}(\mathbb{F})$ and for the whole matrix space $M_{n}(\mathbb{F})$. It is known that for $n=2$, there are bijective linear maps $\Phi$ on $\mathcal{H}_{n}(\mathbb{F})$ and $M_{n}(\mathbb{F})$ satisfying the condition per $A=\operatorname{det} \Phi(A)$. As an application of the obtained results, we show that if $n \geq 3$, then the situation is completely different and already for $n=3$, there is no pair of maps $(\Phi, \phi)$, where $\Phi$ is an arbitrary bijective map on matrices and $\phi: \mathbb{F} \rightarrow \mathbb{F}$ is an arbitrary map such that per $A=\phi(\operatorname{det} \Phi(A))$ for all matrices $A$ from the spaces $\mathcal{H}_{n}(\mathbb{F})$ and $M_{n}(\mathbb{F})$, respectively. Moreover, for the space $M_{n}(\mathbb{F})$, we show that such a pair of transformations does not exist also for an arbitrary $n>3$ if the field $\mathbb{F}$ contains sufficiently many elements (depending on $n$ ). Our results are illustrated by a number of examples.
\end{abstract}

\section{Introduction}

The permanent and the determinant of an $(n \times n)$-matrix $A=\left(a_{i j}\right)$ with real entries $a_{i j}$ are defined by

$$
\text { per } A=\sum_{\sigma \in \mathfrak{S}_{n}} \prod_{i=1}^{n} a_{i \sigma(i)}, \quad \operatorname{det} A=\sum_{\sigma \in \mathfrak{S}_{n}} \operatorname{sgn}(\sigma) \prod_{i=1}^{n} a_{i \sigma(i)},
$$

respectively, where $\mathfrak{S}_{n}$ denotes the symmetric group of all permutations of the set $\{1,2, \ldots, n\}$. The value $\operatorname{sgn}(\sigma) \in\{-1,1\}$ is the sign of the permutation $\sigma$, i.e., $\operatorname{sgn}(\sigma)=1$ if $\sigma$ is an even permutation and $\operatorname{sgn}(\sigma)=-1$ if $\sigma$ is an odd permutation.

The formula for the permanent is similar to, and even simpler than, the formula for the determinant. In the case where the ground field is of characteristic 2, these invariants even coincide. However, in general, there is a polynomial algorithm for calculating the determinant, whereas calculating the permanent is $\sharp P$-complete, as was shown by Valiant [16]. This paradox can be explained by the the fact that there exist many linear transformations that preserve the determinant (see [13]), while there are only a few ones that preserve the permanent (see [12]). Looking at the similarity between the formulas for the permanent and the determinant, Pólya [14] in 1913 asked if it is possible to calculate the permanent using the determinant.

For $n=2$, Pólya observed that it is always possible to convert the permanent to the determinant just by changing the sign of one off-diagonal element. It was soon discovered by Szegő [15] that for any $n \geq 3$, there are $(n \times n)$-matrices for which this Pólya $(2 \times 2)$-argument does not work. This leads to the natural question of when Pólya's approach of changing sign can be used to calculate the permanent of a square matrix, and this is known as the Pólya permanent problem.

The result of Szegö was later generalized in two different directions. On the one hand, the nonexistence of the converters, i.e., such matrix transformations $\Phi$ that per $A=\operatorname{det} \Phi(A)$ for all matrices $A$, was confirmed in different classes of matrix transformations. One of the first results here belongs to Marcus and Minc [12] and asserts that conversion is not possible in the class of linear transformation. Then these investigations were continued in [7-9] (see also the references therein). Moreover, the same problem was considered on various matrix subspaces (see, e.g., $[4,5,10]$ ). On the other hand, the

Translated from Sovremennaya Matematika i Ee Prilozheniya (Contemporary Mathematics and Its Applications), Vol. 80, Proceedings of the International Conference "Modern Algebra and Its Applications" (Batumi, 2011), Part 1, 2012. 
structure of matrices where the permanent is convertible to the determinant by changing signs is also studied (see, e.g., [11]).

We denote by $M_{n}(\mathbb{F})$ the set of $(n \times n)$-matrices with entries from a given field $\mathbb{F}$. Below, unless otherwise explicitly stated, we consider only matrices over a finite field $\mathbb{F}$, whose characteristic $\operatorname{ch} \mathbb{F} \neq 2$ and cardinality $|\mathbb{F}|$ is a power of some prime $p>2$. Let $\mathcal{H}_{n}(\mathbb{F})$ denote the subset of Hermitian matrices (in the beginning of Sec. 2, we recall the definition of a Hermitian matrix over a finite field).

The paper is organized as follows. Section 2 deals with the number of zeros of permanent and determinant functions on the set of Hermitian matrices. As a consequence of the obtained results, we show that the situation for $n>2$ differs from the case $n=2$, and already for $n=3$, there is no pairs $(\Phi, \phi)$ such that per $A=\phi(\operatorname{det} \Phi(A))$ for all matrices $A$ from $\mathcal{H}_{n}(\mathbb{F})$. In Sec. 3, we show that for $n=3$, there are no pairs $(\Phi, \phi)$, where $\Phi: M_{n}(\mathbb{F}) \rightarrow M_{n}(\mathbb{F})$ is bijective and $\phi: \mathbb{F} \rightarrow \mathbb{F}$ is arbitrary, such that per $A=\phi(\operatorname{det} \Phi(A))$ for all matrices $A$ from $M_{n}(\mathbb{F})$. Then for $n>3$, we show that there are no such pairs $(\Phi, \phi)$ for any sufficiently large field.

\section{Permanent Versus Determinant on $\mathcal{H}_{n}$}

Now we describe in detail Hermitian matrices over finite fields. Recall that a complex matrix $A \in M_{n}(\mathbb{C})$ is Hermitian if $A=\bar{A}^{t}$, where the involution $x \rightarrow \bar{x}$ is the complex conjugation. First, we recall the definition of an involution.

Definition 2.1. An involution on a field $\mathbb{F}$ is a map

$$
-: \mathbb{F} \rightarrow \mathbb{F}
$$

that satisfies the following conditions:

(1) $\overline{\bar{x}}=x$,

(2) $\overline{x y}=\bar{y} \bar{x}$ and $\overline{x+y}=\bar{x}+\bar{y}$.

An involution is nonidentical if - is not the identity map.

Note that if a nonidentical involution exists on a field $\mathbb{F}$ of cardinality $p^{m}$, then $m$ is even, and hence $|\mathbb{F}|=q^{2}$, where $q:=p^{m / 2}$. Moreover, in such case the nonidentical involution is unique and is defined by $\bar{x}=x^{q}$ (see the proof of $[6$, Theorem 2]).

Definition 2.2. The set $\mathbb{K}:=\{x \in \mathbb{F}: x=\bar{x}\}$ is called the fixed field of the involution.

It is easy to see that $\mathbb{K}$ is indeed a field, $|\mathbb{K}|=q$, and $\mathbb{F}=\mathbb{K} \oplus \imath \mathbb{K}$ for some $\imath \in \mathbb{F} \backslash \mathbb{K}$ (for detail, see $[2])$.

Note that the map

$$
\mathbb{F} \backslash\{0\} \rightarrow \mathbb{K} \backslash\{0\}, \quad x \mapsto x \bar{x}=x^{q+1},
$$

is surjective and $(q+1)$-to- 1 , that is, for any $\lambda \in \mathbb{K} \backslash\{0\}$, there exist precisely $q+1$ scalars $x \in \mathbb{F}$ such that $x \bar{x}=\lambda$ (for details, see [2, Sec. 2]).

Definition 2.3. Introduce the notation $A^{*}:=\bar{A}^{t}$. Then

$$
\mathcal{H}_{n}(\mathbb{F}):=\left\{A \in \mathcal{M}_{n}(\mathbb{F}): A^{*}=A\right\}
$$

is the set of Hermitian matrices over $\mathbb{F}$.

Lemma 2.4 (see [2]). Any $A \in \mathcal{H}_{n}(\mathbb{F})$ can be written as $A=P\left(E_{11}+\cdots+E_{r r}\right) P^{*}$, where $r:=\operatorname{rk} A$ is its rank. Consequently,

$$
\begin{aligned}
\operatorname{det}(A) & =\operatorname{det}(P) \operatorname{det}\left(P^{*}\right) \operatorname{det}\left(E_{11}+\cdots+E_{r r}\right) \\
& =\operatorname{det}(P) \overline{\operatorname{det}(P)} \operatorname{det}\left(E_{11}+\cdots+E_{r r}\right) \in \mathbb{K} .
\end{aligned}
$$

Note that in the case $n=2$, there exist pairs of transformations $(\Phi, \phi)$ that convert the permanent to the determinant on Hermitian matrices. Such examples exist with the identity or nonidentity map $\phi$. 
Example 2.5. Let $\Phi: \mathcal{H}_{2}(\mathbb{F}) \rightarrow \mathcal{H}_{2}(\mathbb{F})$ be defined by

$$
\Phi(A)=\left(\begin{array}{rr}
a_{11} & \overline{x a_{12}} \\
x a_{12} & a_{22}
\end{array}\right) \text { for } A=\left(\begin{array}{ll}
a_{11} & \overline{a_{12}} \\
a_{12} & a_{22}
\end{array}\right),
$$

where $x \bar{x}=-1$, and let $\phi: \mathbb{K} \rightarrow \mathbb{K}$ be the identity map. Then per $A=\phi(\operatorname{det} \Phi(A))$.

Example 2.6. Let $\Phi: \mathcal{H}_{2}(\mathbb{F}) \rightarrow \mathcal{H}_{2}(\mathbb{F})$ be defined by

$$
\Phi(A)=\left(\begin{array}{rr}
-a_{11} & \overline{a_{12}} \\
a_{12} & a_{22}
\end{array}\right) \quad \text { for } \quad A=\left(\begin{array}{ll}
a_{11} & \overline{a_{12}} \\
a_{12} & a_{22}
\end{array}\right)
$$

and $\phi: \mathbb{K} \rightarrow \mathbb{K}$ be defined by $\phi(\lambda)=-\lambda$ for all $\lambda \in \mathbb{K}$. Then per $A=\phi(\operatorname{det} \Phi(A))$.

Note that in these two examples, $\Phi$ is linear over $\mathbb{K}$ and bijective. If the linearity assumption is removed, one can easily find nonbijective converting maps for the matrices of an arbitrary size.

Example 2.7. Let $\phi: \mathbb{K} \rightarrow \mathbb{K}$ be any bijective transformation and $\Phi: \mathcal{H}_{n}(\mathbb{F}) \rightarrow \mathcal{H}_{n}(\mathbb{F})$ be defined by

$$
\Phi(A)=\operatorname{diag}\left(\phi^{-1}(\operatorname{per} A), 1, \ldots, 1\right) ;
$$

note that per $A \in \mathbb{K}$ by Lemma 2.18 below. Then per $A=\phi(\operatorname{det} \Phi(A))$.

The purpose of this section is to show that if $n \geq 3$, then for bijective $\Phi$, such conversions do not exist.

Below we collect some results on graph theory which will be useful for our considerations.

Let $\Gamma$ be a finite undirected connected graph with the vertex set $V(\Gamma)$, with no multiple edges and loops. Let $\delta(u, v)$ denote the distance between vertices $u$ and $v$, and $\operatorname{diam}(\Gamma)=\max _{u, v \in V(\Gamma)} \delta(u, v)$ denote the diameter of $\Gamma$.

Definition 2.8. The graph $\Gamma$ is distance-regular if there exist numbers $p_{i j}^{k}, 0 \leq i, j, k \leq \operatorname{diam}(\Gamma)$, such that for any $k$ and any pair of vertices $(u, v)$ with $\delta(u, v)=k$ the number of vertices $w$ with $\delta(u, w)=i$ and $\delta(w, v)=j$ equals $p_{i j}^{k}$.

Definition 2.9. Let $\Gamma$ be a distance-regular graph. For any $i, 0 \leq i \leq \operatorname{diam}(\Gamma)$, we denote $b_{i}=p_{1, i+1}^{i}$, $c_{i}=p_{1, i-1}^{i}$, and $k_{i}=p_{i, i}^{0}$.

Lemma 2.10 (see [3, pp. 126-127]). In a distance-regular graph,

$$
k_{0}=1, \quad k_{i+1}=\frac{k_{i} b_{i}}{c_{i+1}} \quad \forall i=0,1, \ldots, \operatorname{diam}(\Gamma)-1 .
$$

Lemma 2.11 (see [3, Theorem 9.5.7]). Let $\Gamma_{\mathcal{H}}$ be a graph with the vertex set $\mathcal{H}_{n}(\mathbb{F})$, where $A, B \in$ $\mathcal{H}_{n}(\mathbb{F})$ are incident on the same edge if and only if $\operatorname{rk}(A-B)=1$. Then $\Gamma_{\mathcal{H}}$ is connected and distance-regular with diameter $n$, the distances here is given by the formula $\delta(A, B)=\operatorname{rk}(A-B)$, and

$$
b_{i}=\frac{q^{2 n}-q^{2 i}}{q+1}, \quad c_{i}=\frac{q^{i-1}\left(q^{i}-(-1)^{i}\right)}{q+1}, \quad i=0,1, \ldots, n .
$$

We proceed by calculating the number of $n \times n$ Hermitian matrices with zero determinant.

Lemma 2.12. Let $\operatorname{det}: \mathcal{H}_{n}(\mathbb{F}) \rightarrow \mathbb{K}$ be the determinant function. Then

$$
\left|\operatorname{det}^{-1}(0)\right|=q^{n^{2}}-\prod_{i=0}^{n-1} \frac{q^{2 n}-q^{2 i}}{q^{i}\left(q^{i+1}+(-1)^{i}\right)} .
$$

Proof. For the graph $\Gamma_{\mathcal{H}}$ from Lemma 2.11 we have by Lemma 2.10 that

$$
k_{n}=k_{0} \prod_{i=0}^{n-1} \frac{b_{i}}{c_{i+1}} \text {. }
$$


Then substituting the values of $k_{0}, b_{i}, c_{i}$ evaluated in Lemma 2.11, we obtain

$$
k_{n}=k_{0} \prod_{i=0}^{n-1} \frac{b_{i}}{c_{i+1}}=\prod_{i=0}^{n-1} \frac{q^{2 n}-q^{2 i}}{q^{i}\left(q^{i+1}+(-1)^{i}\right)} .
$$

By Lemma 2.11, $\operatorname{rk}(A)=n$ if and only if $\delta(0, A)=n$, so $k_{n}$ equals the number of all invertible Hermitian matrices. Hence, the proof ends by the equalities

$$
\left|\operatorname{det}^{-1}(0)\right|=\left|\mathcal{H}_{n}(\mathbb{F})\right|-k_{n}, \quad\left|\mathcal{H}_{n}(\mathbb{F})\right|=q^{n^{2}} .
$$

Corollary 2.13. If $n=3$, then

$$
\left|\operatorname{det}^{-1}(0)\right|=q^{8}-q^{7}+2 q^{6}-q^{5}+q^{4}-q^{3} .
$$

Given a nonzero column vector $\boldsymbol{x} \in \mathbb{F}^{n}$, let $\langle\boldsymbol{x}\rangle$ denote the one-dimensional vector space that is spanned by $\boldsymbol{x}$.

Definition 2.14. Let $C \in \mathcal{H}_{n}(\mathbb{F})$ be invertible. The set of all vector spaces $\langle\boldsymbol{x}\rangle$ that satisfy $\boldsymbol{x}^{t} C \overline{\boldsymbol{x}}=0$ is called the Hermitian variety of $C$.

In order to estimate the number of $(n \times n)$-matrices with zero permanent we need the following technical lemma.

Lemma 2.15. [2, Theorem 8.1] Let $C$ be an invertible Hermitian matrix. Then its variety has cardinality

$$
\frac{\left(q^{n}-(-1)^{n}\right)\left(q^{n-1}-(-1)^{n-1}\right)}{q^{2}-1} .
$$

Definition 2.16. Let $\mathcal{S}$ be some system of equations and inequalities in $\mathbb{F}^{n}$. The symbol $N(\mathcal{S})$ denotes the number of all solutions of the system $\mathcal{S}$ in $\mathbb{F}^{n}$.

Lemma 2.17. Let $C \in \mathcal{H}_{n}(\mathbb{F})$ be invertible,

$$
\sum_{i, j} c_{i j} x_{i} \bar{x}_{j}=\boldsymbol{x}^{t} C \overline{\boldsymbol{x}}
$$

and $c_{i j}=\bar{c}_{j i}$ be a Hermitian form corresponding to the matrix $C$. Then

$$
N\left(\boldsymbol{x}^{t} C \overline{\boldsymbol{x}}=0\right)=q^{2 n-1}+(-1)^{n}(q-1) q^{n-1} .
$$

Proof. Any nonzero multiple of a nonzero column vector $\boldsymbol{x}$ spans the same space $\langle\boldsymbol{x}\rangle$. Hence, to calculate the number of nonzero solutions of $\boldsymbol{x}^{t} C \overline{\boldsymbol{x}}=0$, we need to multiply (1) by $|\mathbb{F} \backslash\{0\}|=q^{2}-1$. Then to find $N\left(\boldsymbol{x}^{t} C \overline{\boldsymbol{x}}=0\right)$ we add 1 to the result, counting the zero column vector.

Lemma 2.18. Let $\mathbb{F}$ be a finite field with a nonidentical involution. Let $A=\left[a_{i j}\right]$ be an $n \times n$ Hermitian matrix. Then per $A \in \mathbb{K}$.

Proof. Observe that

$$
a_{1 \sigma^{-1}(1)} a_{2 \sigma^{-1}(2)} \cdots a_{n \sigma^{-1}(n)}=a_{\sigma(1) 1} a_{\sigma(2) 2} \cdots a_{\sigma(n) n}
$$

for each permutation $\sigma$ of the set $\{1,2, \ldots, \mathrm{n}\}$. Hence,

$$
\operatorname{per} A=\sum_{\sigma} a_{1 \sigma^{-1}(1)} a_{2 \sigma^{-1}(2)} \cdots a_{n \sigma^{-1}(n)}=\sum_{\sigma} a_{\sigma(1) 1} a_{\sigma(2) 2} \cdots a_{\sigma(n) n}=\operatorname{per} A^{t}=\operatorname{per} \bar{A}=\overline{\operatorname{per} A} .
$$

Lemma 2.19. Let $\mathbb{F}$ be a finite field with a nonidentical involution. The functions

$$
\lambda \mapsto\left|\operatorname{det}^{-1}(\lambda) \cap \mathcal{H}_{n}(\mathbb{F})\right|, \quad \lambda \mapsto\left|\operatorname{per}^{-1}(\lambda) \cap \mathcal{H}_{n}(\mathbb{F})\right|
$$

are constant for nonzero $\lambda \in \mathbb{K}$. 
Proof. Choose any nonzero $\mu \in \mathbb{F}$. It induces a bijection

$$
A \mapsto\left(\mu \oplus \operatorname{Id}_{n-1}\right) A\left(\mu \oplus \operatorname{Id}_{n-1}\right)^{*}
$$

on $\mathcal{H}_{n}(\mathbb{F})$. Moreover, by using the Laplace decomposition twice in succession starting with the first row and then continuing on the first column,

$$
\operatorname{per}\left(\left(\mu \oplus \operatorname{Id}_{n-1}\right) A\left(\mu \oplus \operatorname{Id}_{n-1}\right)^{*}\right)=\mu \bar{\mu} \operatorname{per}(A)
$$

and likewise for determinant. So this map induces a bijection between the sets

$$
\operatorname{per}^{-1}(1) \cap \mathcal{H}_{n}(\mathbb{F}) \text { and } \operatorname{per}^{-1}(\mu \bar{\mu}) \cap \mathcal{H}_{n}(\mathbb{F}) .
$$

Hence,

$$
\left|\operatorname{per}^{-1}(1) \cap \mathcal{H}_{n}(\mathbb{F})\right|=\left|\operatorname{per}^{-1}(\mu \bar{\mu}) \cap \mathcal{H}_{n}(\mathbb{F})\right|
$$

and likewise

$$
\left|\operatorname{det}^{-1}(1) \cap \mathcal{H}_{n}(\mathbb{F})\right|=\left|\operatorname{det}^{-1}(\mu \bar{\mu}) \cap \mathcal{H}_{n}(\mathbb{F})\right|
$$

for any nonzero $\mu \in \mathbb{F}$. This completes the proof, since any nonzero $\lambda \in \mathbb{K}$ is of the form $\lambda=\mu \bar{\mu}$ for a suitable $\mu \in \mathbb{F}$.

Lemma 2.20. Let $q$ be odd, $\mathbb{F}$ be a field, $|\mathbb{F}|=q^{2}$, and per : $\mathcal{H}_{3}(\mathbb{F}) \rightarrow \mathbb{K}$ be the permanent function. Then

$$
\left|\operatorname{per}^{-1}(0)\right|=q^{8}+q^{6}-3 q^{5}+3 q^{4}-q^{2}
$$

Proof. By definition,

$$
\operatorname{per}\left(\left[\begin{array}{lll}
a & \bar{b} & \bar{c} \\
b & d & \bar{e} \\
c & e & f
\end{array}\right]\right)=a(d f+e \bar{e})+f \cdot b \bar{b}+(e \cdot b \bar{c}+\bar{e} \cdot \bar{b} c)+d \cdot c \bar{c} .
$$

Note that $a, d, f \in \mathbb{K}$ and $b, c, e \in \mathbb{F}$, where $|\mathbb{K}|=q$ and $|\mathbb{F}|=q^{2}$. Hence

$$
\begin{array}{r}
N\left(\operatorname{per}\left(\left[\begin{array}{lll}
a & \bar{b} & \bar{c} \\
b & d & \bar{e} \\
c & e & f
\end{array}\right]\right)=0\right) \\
=N(d f+e \bar{e} \neq 0) \cdot\left(q^{2}\right)^{2}+N(d f+e \bar{e}=0, f \cdot b \bar{b}+(e \cdot b \bar{c}+\bar{e} \cdot \bar{b} c)+d \cdot c \bar{c}=0) \cdot q \\
=N(d f+e \bar{e} \neq 0) \cdot q^{4}+N(d f+e \bar{e}=0, d f-e \bar{e}=0, f \cdot b \bar{b}+(e \cdot b \bar{c}+\bar{e} \cdot \bar{b} c)+d \cdot c \bar{c}=0) \cdot q \\
+N(d f+e \bar{e}=0, d f-e \bar{e} \neq 0, f \cdot b \bar{b}+(e \cdot b \bar{c}+\bar{e} \cdot \bar{b} c)+d \cdot c \bar{c}=0) \cdot q
\end{array}
$$

Now

$$
N(d f+e \bar{e} \neq 0)=q \cdot q \cdot q^{2}-N(d f+e \bar{e}=0) .
$$

Since the map

$$
\mathbb{F} \backslash\{0\} \rightarrow \mathbb{K} \backslash\{0\}, \quad x \mapsto x \bar{x},
$$

is $(q+1)$-to- 1 , it is easy to see that

$$
N(d f+e \bar{e}=0)=(q-1)^{2}(q+1)+(2 q-1)=q^{3}-q^{2}+q .
$$

Consequently,

$$
N(d f+e \bar{e} \neq 0)=q^{4}-q^{3}+q^{2}-q .
$$

To calculate

$$
N(d f+e \bar{e}=0, d f-e \bar{e}=0, f \cdot b \bar{b}+(e \cdot b \bar{c}+\bar{e} \cdot \bar{b} c)+d \cdot c \bar{c}=0)
$$


note that the considered equations are satisfied precisely in the following three cases:

$$
\begin{aligned}
& e=0, d=0=f, b \text { and } c \text { are arbitrary, } \\
& e=0, d=0, f \neq 0, b=0, c \text { is arbitrary, } \\
& e=0, d \neq 0, f=0, c=0, b \text { is arbitrary. }
\end{aligned}
$$

Hence

$$
N(d f+e \bar{e}=0, d f-e \bar{e}=0, f \cdot b \bar{b}+(e \cdot b \bar{c}+\bar{e} \cdot \bar{b} c)+d \cdot c \bar{c}=0)=\left(q^{2}\right)^{2}+2(q-1) q^{2} .
$$

Finally, we calculate

$$
N(d f+e \bar{e}=0, d f-e \bar{e} \neq 0, f \cdot b \bar{b}+(e \cdot b \bar{c}+\bar{e} \cdot \bar{b} c)+d \cdot c \bar{c}=0) .
$$

First, note that

$$
f \cdot b \bar{b}+(e \cdot b \bar{c}+\bar{e} \cdot \bar{b} c)+d \cdot c \bar{c}=(b, c)\left[\begin{array}{ll}
f & e \\
\bar{e} & d
\end{array}\right] \overline{(b, c)}^{t},
$$

where the determinant of the matrix is $d f-e \bar{e} \neq 0$. Hence, if a tuple $(d, e, f)$ satisfies

$$
d f+e \bar{e}=0, \quad d f-e \bar{e} \neq 0,
$$

then, by Lemma 2.17, there exist $q^{3}+q^{2}-q$ tuples $(b, c)$ with

$$
f \cdot b \bar{b}+(e \cdot b \bar{c}+\bar{e} \cdot \bar{b} c)+d \cdot c \bar{c}=0 .
$$

Since

$$
\begin{aligned}
N(d f+e \bar{e}=0, d f-e \bar{e} & \neq 0) \\
& =N(d f+e \bar{e}=0)-N(d f+e \bar{e}=0, d f-e \bar{e}=0) \\
& =q^{3}-q^{2}+q-(2 q-1)=q^{3}-q^{2}-q+1,
\end{aligned}
$$

we deduce that

$$
\begin{aligned}
N(d f+e \bar{e}=0, d f-e \bar{e} \neq 0, f \cdot b \bar{b}+(e \cdot b \bar{c}+\bar{e} \cdot \bar{b} c)+d \cdot c \bar{c} & =0) \\
& =\left(q^{3}-q^{2}-q+1\right) \cdot\left(q^{3}+q^{2}-q\right) .
\end{aligned}
$$

Now substituting Eqs. (3), (4), and (5) into formula (2), after the necessary simplifications we obtain the desired formula.

Theorem 2.21. Let $n=3$. Then then there exists no pair of maps $(\Phi, \phi)$, with

$$
\Phi: \mathcal{H}_{n}(\mathbb{F}) \rightarrow \mathcal{H}_{n}(\mathbb{F}), \quad \phi: \mathbb{K} \rightarrow \mathbb{K}
$$

such that $\Phi$ is bijective and

$$
\operatorname{per} A=\phi(\operatorname{det} \Phi(A)) ; \quad A \in \mathcal{H}_{n}(\mathbb{F}) .
$$

Proof. It follows directly from Corollary 2.13 and Lemma 2.20 that

$$
\left|\operatorname{per}^{-1}(0)\right|-\left|\operatorname{det}^{-1}(0)\right|=(q+1)^{2} q^{2}(q-1)^{3},
$$

so there are more $3 \times 3$ Hermitian matrices with zero permanent than with zero determinant, and thus there is no such $\Phi$ in the case $\phi(0)=0$.

By Lemma 2.19 we have that

$$
\begin{aligned}
\left|\operatorname{det}^{-1}(\lambda)\right|=\left(q^{9}-q^{8}+q^{7}-2 q^{6}+q^{5}-q^{4}+q^{3}\right) /(q-1) & \\
& =(q-1) q^{3}\left(q^{2}+1\right)\left(q^{2}+q+1\right) \quad \forall \lambda \in \mathbb{K} \backslash\{0\} .
\end{aligned}
$$

Similarly,

$$
\left|\operatorname{per}^{-1}(\lambda)\right|=\left(q^{9}-q^{8}-q^{6}+3 q^{5}-3 q^{4}+q^{2}\right) /(q-1) \quad \forall \lambda \in \mathbb{K} \backslash\{0\}
$$


Note that per $: \mathcal{H}_{3}(\mathbb{F}) \rightarrow \mathbb{K}$ is a surjective map since the image of the permanent lies in $\mathbb{K}$ by Lemma 2.18, and diagonal matrices from $\mathcal{H}_{3}(\mathbb{F})$ have entries from $\mathbb{K}$ on the diagonal, so

$$
\operatorname{per}(\operatorname{diag}(\alpha, 1,1))=\alpha \quad \forall \alpha \in \mathbb{K} .
$$

Now, if the required pair $(\Phi, \phi)$ does exists, then $\phi$ is surjective since $\Phi$ is bijective and per is surjective. Then $\phi$ is bijective since $\mathbb{K}$ is finite. Hence, by the aforesaid, for some nonzero $\alpha, \Phi$ maps the set $\operatorname{per}^{-1}(\alpha)$ bijectively onto $\operatorname{det}^{-1}(0)$. Since $|\mathbb{F}|>2$, there exists $\beta \in \mathbb{F} \backslash\{0\}$ such that $\Phi$ maps $\operatorname{per}^{-1}(\beta)$ to $\operatorname{det}^{-1}(\mu)$ for some $\mu \neq 0$. Due to the relation

$$
\left|\operatorname{per}^{-1}(\alpha)\right|=\left|\operatorname{per}^{-1}(\beta)\right|,
$$

the bijective $\Phi$ requires that

$$
q^{3}\left(\left(q^{4}+q^{2}+1\right)(q-1)+q^{3}\right)=\left|\operatorname{det}^{-1}(0)\right|=\left|\operatorname{det}^{-1}(\mu)\right|=(q-1) q^{3}\left(q^{2}+1\right)\left(q^{2}+q+1\right)
$$

for some nonzero $\beta \in \mathbb{F}$. This is a contradiction because the right-hand side is divisible by an integer $q-1$ but the left-hand side is not.

\section{Permanent Versus Determinant on $M_{n}(\mathbb{F})$}

In this section, $|\mathbb{F}|=q$.

Recall that in 1913 Pólya discovered (see [14]) that in the case $n=2$ the following linear transformation converts the permanent to the determinant.

Example 3.1. Let $\Phi: M_{2}(\mathbb{F}) \rightarrow M_{2}(\mathbb{F})$ be defined as follows:

$$
\text { for } A=\left(\begin{array}{ll}
a_{11} & a_{12} \\
a_{21} & a_{22}
\end{array}\right) \text {, one has } \Phi(A)=\left(\begin{array}{rr}
a_{11} & -a_{12} \\
a_{21} & a_{22}
\end{array}\right) \text {. }
$$

Then $\operatorname{per} A=\operatorname{det} \Phi(A)$.

The following result on the absence of conversion for the permanent and the determinant over $M_{n}(\mathbb{F})$, where $\mathbb{F}$ is a finite field, $n \geq 3$, was proved in [7].

Theorem 3.2 (see [7, Theorem 2.1]). Let $n \geq 3$ and let $\mathbb{F}$ be a finite field with $\operatorname{ch} \mathbb{F} \neq 2$ and with sufficiently large cardinality (which depends only on $n$ ). Then no bijective map $T: M_{n}(\mathbb{F}) \rightarrow M_{n}(\mathbb{F})$ satisfies per $A=\operatorname{det} T(A)$.

The following question arises naturally after the above result is obtained.

Question 3.3. Can we utilize simultaneously two mappings

$$
\Phi: M_{n}(\mathbb{F}) \rightarrow M_{n}(\mathbb{F}), \quad \phi: \mathbb{F} \rightarrow \mathbb{F}
$$

such that per $A=\phi(\operatorname{det} \Phi(A))$ ?

Note that in Example 3.1 map $\phi$ is the identical map.

Below we show that already for $n=3$ the situation is quite different from Example 3.1: not only is the conversion impossible, but there is actually no pair of transformations $(\Phi, \phi)$, where $\Phi: M_{n}(\mathbb{F}) \rightarrow$ $M_{n}(\mathbb{F})$ is bijective and $\phi: \mathbb{F} \rightarrow \mathbb{F}$ is arbitrary, such that

$$
\operatorname{per} A=\phi(\operatorname{det} \Phi(A)) \text {. }
$$

Theorem 3.4. Let $\mathbb{F}$ be a finite filed, ch $\mathbb{F} \neq 2$. Then there exists no pair of maps $\left(\Phi: M_{3}(\mathbb{F}) \rightarrow\right.$ $\left.M_{3}(\mathbb{F}), \phi: \mathbb{F} \rightarrow \mathbb{F}\right)$, where $\Phi$ is bijective, such that per $A=\phi(\operatorname{det} \Phi(A))$. 
Proof. Step 1. It was shown in [7, Lemma 3.1] that

$$
\left|P_{3}(\mathbb{F})\right|=\left|D_{3}(\mathbb{F})\right|-q^{2}(q-1)^{5},
$$

where $P_{3}(\mathbb{F})$ and $D_{3}(\mathbb{F})$ denote the set of $(3 \times 3)$-matrices with zero permanent and zero determinant, respectively. By [1, Proposition 2, p. 41], we obtain

$$
\left|D_{3}(\mathbb{F})\right|=q^{9}-\left(q^{3}-q^{2}\right)\left(q^{3}-q\right)=q^{8}+q^{7}-q^{5}-q^{4}+q^{3},
$$

and so

$$
\left|P_{3}(\mathbb{F})\right|=\left(q^{8}+q^{7}-q^{5}-q^{4}+q^{3}\right)-q^{2}(q-1)^{5}=q^{8}+5 q^{6}-11 q^{5}+9 q^{4}-4 q^{3}+q^{2} .
$$

Step 2. By [7, Lemma 7.1], the number of matrices with the determinant (respectively, permanent) $\lambda$ is equal to the number of matrices with the determinant (respectively, permanent) $\mu$ for any $\lambda, \mu \in$ $\mathbb{F} \backslash\{0\}$. Hence

$$
\left|\operatorname{det}^{-1}(\lambda)\right|=\frac{q^{9}-\left|D_{3}(\mathbb{F})\right|}{q-1}=(q-1)^{2} q^{2}\left(q^{4}+2 q^{3}+2 q^{2}+q\right) \quad \forall \lambda \in \mathbb{F} \backslash\{0\}
$$

and

$$
\left|\operatorname{per}^{-1}(\lambda)\right|=\frac{q^{9}-\left|P_{3}(\mathbb{F})\right|}{q-1}=(q-1)^{2} q^{2}\left(q^{4}+2 q^{3}+3 q^{2}-q+1\right) \quad \forall \lambda \in \mathbb{F} \backslash\{0\} .
$$

Step 3. Now we are ready to show that there is no a pair $(\Phi, \phi)$, where $\Phi$ is bijective, such that $\operatorname{per} A=\phi(\operatorname{det} \Phi(A))$.

Indeed, if such a pair does exists, then $\phi$ is surjective since $\Phi$ is bijective and per is surjective. Then $\phi$ is bijective since $\mathbb{F}$ is finite. Hence $\Phi$ maps the set $\operatorname{per}^{-1}(0)$ bijectively onto one of the sets $\operatorname{det}^{-1}(\lambda)$, $\lambda \in \mathbb{F}$. By Step $1, \lambda \neq 0$. Since $|\mathbb{F}|>2$, there exists $\alpha \in \mathbb{F} \backslash\{0\}$ such that $\Phi$ maps $\operatorname{per}^{-1}(\alpha)$ to $\operatorname{det}^{-1}(\mu)$ for some $\mu \neq 0$. Then

$$
\left|\operatorname{per}^{-1}(0)\right|=\left|\operatorname{det}^{-1}(\lambda)\right|
$$

and

$$
\left|\operatorname{per}^{-1}(\alpha)\right|=\left|\operatorname{det}^{-1}(\mu)\right|
$$

since $\Phi$ is bijective. The right-hand sides of these equalities are equal to each other by the formula (10). Hence, the left-hand sides of these inequalities are equal, i.e.,

$$
\left|\operatorname{per}^{-1}(0)\right|=\left|\operatorname{per}^{-1}(\alpha)\right| \text {. }
$$

The last equality contradicts formulas (9) and (11). Namely, straightforward numerical calculations show that, other than $q=0$, no solution of the polynomial equation $(9)=(11)$ is an integer.

Next we show that the situation with conversion is similar for $n>3$.

Theorem 3.5. Assume that $n>3$. Then there exists $q_{0}$, depending on $n$, such that for any finite field $\mathbb{F}$ with at least $q_{0}$ elements and $\operatorname{ch} \mathbb{F} \neq 2$, there exists no pair of maps $\left(\Phi: M_{n}(\mathbb{F}) \rightarrow M_{n}(\mathbb{F}), \phi: \mathbb{F} \rightarrow \mathbb{F}\right)$, with $\Phi$ bijective, such that

$$
\operatorname{per} A=\phi(\operatorname{det} \Phi(A)) .
$$

Proof. On the contrary, we assume that such a pair $(\Phi, \phi)$ exists. Since per $A$ is a surjective function onto $\mathbb{F}$, the transformation $\phi$ must be surjective. Hence $\phi$ is also bijective, since $\mathbb{F}$ is finite.

Note that $\phi(0) \neq 0$, otherwise $\Phi$ maps the set per ${ }^{-1}(0)$ bijectively onto $\operatorname{det}^{-1}(0)$, contradicting the fact that they have different cardinalities (see [7, Theorem 3.1]. Then, by the surjectivity, we must have $\phi(0)=\alpha$ for some nonzero $\alpha$. Also, because $|\mathbb{F}| \geq 3$, there exist $\beta, \beta^{\prime} \in \mathbb{F} \backslash\{0\}$ such that $\phi\left(\beta^{\prime}\right)=\beta$. Then $\Phi$ would map the sets $\operatorname{per}^{-1}(\alpha)$ and $\operatorname{per}^{-1}(\beta)$ bijectively onto $\operatorname{det}^{-1}(0)$ and $\operatorname{det}^{-1}\left(\beta^{\prime}\right)$ respectively. From [7, Lemma 7.1] we have that

$$
\left|\operatorname{per}^{-1}(\alpha)\right|=\left|\operatorname{per}^{-1}(\beta)\right|
$$


which would via bijection $\Phi$ imply that also

$$
q^{n^{2}}-\prod_{k=1}^{n}\left(q^{n}-q^{k-1}\right)=\left|\operatorname{det}^{-1}(0)\right|=\left|\operatorname{det}^{-1}(\beta)\right|=\frac{\prod_{k=1}^{n}\left(q^{n}-q^{k-1}\right)}{q-1}
$$

and hence

$$
q^{n^{2}}-\prod_{k=1}^{n}\left(q^{n}-q^{k-1}\right)=\frac{\prod_{k=1}^{n}\left(q^{n}-q^{k-1}\right)}{q-1}
$$

Hence, after rearranging,

$$
\prod_{k=1}^{n}\left(q^{n}-q^{k-1}\right)=q^{n^{2}-1}(q-1) .
$$

This is a contradiction, since at fixed $q$, the left side is divisible by an integer

$$
q^{n}-1=(q-1)\left(q^{n-1}+q^{n-2}+\cdots+1\right),
$$

but the right side is not.

Acknowledgements. This research was partially supported by a joint Slovene-Russian grant BIRU/08-09-009. The research of the second author is also supported by the Russian Foundation for Basic Research (project No. 09-01-00303a).

\section{REFERENCES}

1. J. L. Alperin and R. B. Bell, Groups and Representations, Springer-Verlag, New York (1995).

2. R. C. Bose and I. M. Chakravarti, "Hermitian varieties in a finite projective space $\mathrm{PG}\left(N, q^{2}\right)$," Can. J. Math., 18, 1161-1182 (1966).

3. A. E. Brouwer, A. M. Cohen, and A. Neumaier, Distance-Regular Graphs, Ergeb. Math. Grenz. (3), 18, Springer-Verlag, Berlin (1989).

4. M. P. Coelho and M. A. Duffner, "On the conversion of an immanant into another on symmetric matrices," Linear Multilinear Algebra, 51, No. 2, 137-145 (2003).

5. M. P. Coelho and M. A. Duffner, "Subspaces where an immanant is convertible into its conjugate," Linear Multilinear Algebra, 48, No. 4, 383-408 (2001).

6. S. Crvenković, I. Dolinka, and M. Vinčić, "On subdirect decomposition and varieties of some rings with involution," Beiträge Algebra Geom., 43, No. 2, 423-432 (2002).

7. G. Dolinar, A. Guterman, B. Kuzma, and M. Orel, "On the Pólya permanent problem over finite fields, Eur. J. Combin., 32, No. 1, 116-132 (2011).

8. J. von zur Gathen, "Permanent and determinant," Linear Algebra Appl., 96, 87-100 (1987).

9. B. Kuzma, "On immanant-preserving mappings," Fundam. Prikl. Mat., 13, No. 4, 113-120 (2007).

10. M. H. Lim, "A note on the relation between the determinant and the permanent," Linear Multilinear Algebra, 7, No. 2, 145-147 (1979).

11. C. H. C. Little, "A characterization of convertible (0,1)-matrices," J. Combin. Theory, Ser. B, 18, 187-208 (1975).

12. M. Marcus and H. Minc, "On the relation between the determinant and the permanent," Ill. J. Math., 5, 376-381 (1961).

13. M. Marcus and B. Moyls, "Linear transformations on algebras of matrices, Can. J. Math., 11, 61-66 (1959).

14. G. Pólya, "Aufgabe 424," Arch. Math. Phys., 20, No. 3, 271 (1913). 
15. G. Szegö, "Lösungzu 424," Arch. Math. Phys., 21, 291-292 (1913).

16. L. G. Valiant, "The complexity of computing the permanent," Theor. Comput. Sci., 8, 189-201 (1979).

G. Dolinar

University of Ljubljana, Ljubljana, Slovenia

E-mail: gregor.dolinar@fe.uni-lj.si

A. Guterman

Moscow State University, Moscow, Russia

E-mail: guterman@list.ru

B. Kuzma

University of Primorska, Koper, Slovenia;

IMFM, Ljubljana, Slovenia

E-mail: bojan.kuzma@pef.upr.si

M. Orel

University of Primorska, Koper, Slovenia

E-mail: marko.orel@upr.si 